\title{
Scenario Approach for Assessing the Utility of Dispersal Information in Decision Support for Aerially Spread Plant Pathogens, Applied to Phytophthora infestans
}

\author{
P. Skelsey, W. A. H. Rossing, G. J. T. Kessel, and W. van der Werf
}

First and fourth authors: Wageningen University, Department of Plant Sciences, Crop and Weed Ecology Group, P.O. Box 430, 6700 AK Wageningen, The Netherlands; second author: Wageningen University, Department of Plant Sciences, Biological Farming Systems Group, P.O. Box 4563, 6700 AN Wageningen, The Netherlands; and third author: Plant Research International, P.O. Box 16, 6700 AA Wageningen, The Netherlands.

Current address of first author: Evolutionary Genetics Group, University of Groningen, P.O. Box 14, 9750 AA Haren, The Netherlands. Accepted for publication 23 February 2009.

\section{ABSTRACT}

Skelsey, P., Rossing, W. A. H., Kessel, G. J. T., and van der Werf, W. 2009. Scenario approach for assessing the utility of dispersal information in decision support for aerially spread plant pathogens, applied to Phytophthora infestans. Phytopathology 99:887-895.

Opportunities exist to improve decision support systems through the use of dispersal information gained from epidemiological research. However, dispersal and demographic information is often fragmentary in plant pathology, and this uncertainty creates a risk of inappropriate action whenever such information is used as a basis for decision making. In this article, a scenario-based simulation approach is used to evaluate crop and economic risks and benefits in the use of dispersal information for decision making using the potato late blight pathosystem (Phytophthora infestansSolanum tuberosum) as a case study. A recently validated spatiotemporal potato late blight model was coupled to submodels for crop growth, tuber dry matter production, and fungicide efficacy. The yield response of a range of management scenarios to a single influx of primary inoculum (the initial spore load) was calculated. Damage curves (relative yield loss versus initial spore load) from a range of combinations of varietal susceptibility and fungicide treatments were used to classify the various management scenarios as either sensitive to initial spore load or tolerant to initial spore load, thus identifying where a high degree of accuracy would be required in dispersal information for appropriate decision making, and where a greater degree of uncertainty could be tolerated. General epidemics, resulting from spatially homogeneous initial spore loads, responded more strongly to the size of the initial spore load than focal epidemics, resulting from an initial spot infection. Susceptible cultivars responded with sizeable yield losses even at low levels of initial spore load, regardless of the fungicide management regime used. These results indicated that, for susceptible cultivars (late cultivars in particular), the degree of accuracy that would be required in dispersal information for appropriate decision making is unlikely to be practically attainable. The results also indicated that, contrary to "folk wisdom," spore loads of a few hundred spores per square meter do not lead to appreciable crop loss in resistant cultivars and are therefore acceptable. We conclude that scope exists for including dispersal information in decision making for potato late blight with resistant potato cultivars but not for susceptible cultivars. The modeling framework used in this study can be extended to investigate the scope for inclusion of dispersal information in decision support for other aerially transmitted pathogens.

Additional keywords: crop loss assessment, dispersal models.
The study of botanical epidemics has burgeoned over the last few decades, in part because of the many costs attributed to plant disease. Crop loss assessment-more specifically, the study of reduction in quantity of yield in relation to disease development-has accordingly become a common theme in plant pathology. An interest in crop losses is not new, however. Van der plank (21) clearly identified the importance of the disease-yield relationship in evaluating the consequences of plant disease epidemics, and recent decades have seen the formulation of many models of the underlying processes $(4,9,12,19)$. Most commonly studied is the relationship between disease intensity (e.g., severity, area under the disease progress curve) and yield. Less common is to relate primary inoculum to yield. The reason for doing so is intuitive; it is the exponential part of epidemic progress that is relevant for disease management, and this early phase is strongly linked to initial levels of disease and inoculum. Nonetheless, despite the documented evidence that dispersal of inoculum is a key factor in many plant disease epidemics, knowledge of disper-

Corresponding author: P. Skelsey; E-mail address: p.skelsey@rug.nl

doi:10.1094/PHYTO-99-7-0887

(C) 2009 The American Phytopathological Society sal has scarcely been used in the development of practical decision support systems (DSSs) (10). This is due, in part, to the difficulty of collecting empirical data and the mathematical complexity of dispersal models but more to prediction uncertainties stemming from the stochastic nature of dispersal processes. These are a result of the idiosyncrasies of the weather and the difficulty of knowing the locations of disease sources. Therefore, dispersal and demography information is often fragmentary in plant pathology, creating a risk of inappropriate action whenever such information is used as a basis for decision making.

It is a common perception among many farmers and farm advisors that the economic threat posed by certain aerially transmitted plant diseases (e.g., potato late blight [causal agent, Phytophthora infestans]) is so great that no disease or infection risk at all should be tolerated. "Folk wisdom" often regards a single spore as enough to destroy a crop, but is this really the case? The damage that can be caused by arriving inoculum depends on complex interactions between source and target characteristics and environmental conditions. Key factors affecting the arrival of inoculum at a target crop include the size, strength, and location of sources and the weather conditions that affect the release, escape, transport, deposition, and survival of inoculum. Key factors affecting the degree of damage caused by this inocu- 
lum include the amount and spatial distribution of arriving spores, weather conditions, cultivar properties, and the level of chemical protection at the target crop. Therefore, a large number of field experiments would be required to define the relationship between primary inoculum and yield loss but epidemiological models are well suited to investigate the interaction of these risk factors.

The objective of this article is to provide a simulation approach that can be used to assess ex ante whether dispersal information can add value to practical DSSs for aerially transmitted pathogens. This is done by first focusing on the question of how risk from aerially transmitted pathogens depends on the amount of primary inoculum (the initial spore load) that is deposited on the target crop, using potato late blight as a model pathosystem. Risk is assessed in a series of steps. First, the expected epidemic development after deposition of a range of spore loads is calculated. Epidemic development depends on the weather, the resistance level of the cultivar, the fungicide regime, and the spatial distribution of initial inoculum: spot (focal epidemic) or homogeneous (general epidemic). Yield reduction is then calculated by considering the loss of light interception on healthy green foliage throughout the duration of the crop. Therefore, a distinction is drawn between early- and late-maturing cultivars, because they differ substantially in seasonal patterns of light interception and yield formation; whereas early-maturing cultivars have a growing season of only 3 to 4 months, after which the foliage senesces naturally, late-maturing cultivars grow for up to 7 months, giving a disease epidemic much more time to claim the foliage and cause damage (8). Thus, we are able to highlight the yield response to a range of spore inputs for a given choice of cultivar resistance, maturity type, chemical protection scheme, and spatial distribution of initial spore load.

Once yield reduction data are determined for a range of management scenarios, we then focus on the question of how the scope for inclusion of dispersal information in practical DSSs depends on the potential consequences of uncertainty in that dispersal information. This is achieved by presenting yield reduction data in the form of "damage curves." Normally, a damage curve is a graph of yield (or loss) versus disease (11). In this

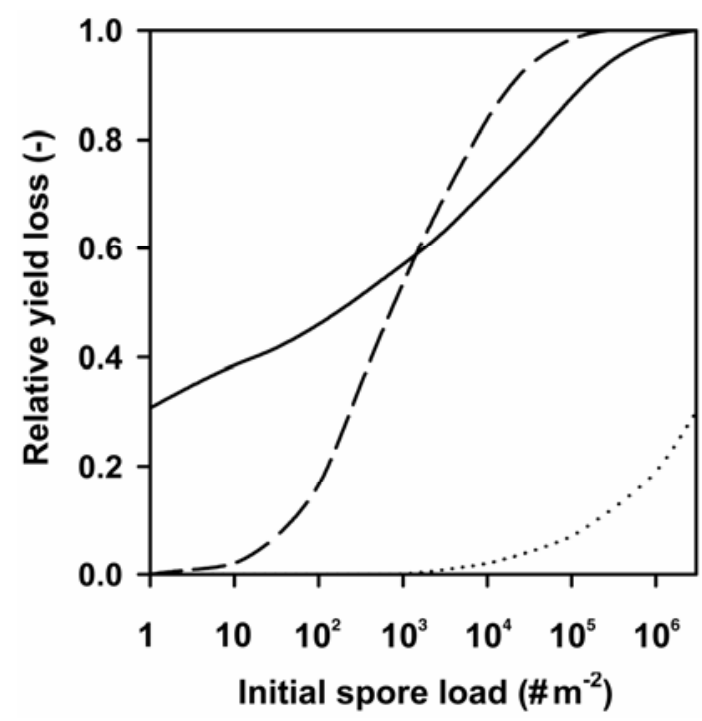

Fig. 1. Simple classification scheme for plant disease management strategies based upon the shape of damage curves. Damage curves show the relationship between yield loss (relative to final yield in a disease-free crop) and primary inoculum (initial spore load) in simulated potato late blight epidemics. All epidemics were initiated with a single influx of spores. The solid line shows a sensitive response to initial spore load, because the damage curve has a large initial step. The dashed line also shows a sensitive response to initial spore load; there is no large initial step but the slope of the curve is steep. The dotted line shows a tolerant response to initial spore load, because the damage curve is initially flat over a wide range of spore loads. study, we define a damage curve as a graph of relative yield loss versus initial spore load. The basic shape of the damage curve is used to indicate where a high degree of accuracy would be required in dispersal information and when a greater degree of uncertainty could be tolerated (Fig. 1). When the damage curve is steep, or has a discontinuity (step), uncertainties in dispersal information could result in inappropriate management decisions, with sizeable consequences. When the damage curve is initially flat or very shallow, the risk from aerially transmitted pathogens is low, the consequences that may arise from uncertainties in dispersal information and subsequent inappropriate management decisions are minimal, and scope exists for inclusion of dispersal information in decision-making. Thus, using a scenario-based approach, the risk from aerially transmitted pathogens can be determined for a given choice of cultivar resistance, maturity type, chemical protection scheme, and spatial distribution of initial spore load, and management conditions can be identified where there is scope for the inclusion of dispersal information in decision making.

\section{MATERIALS AND METHODS}

The spatiotemporal/integrodifference equation model of the potato late blight pathosystem developed by Skelsey et al. (17) simulates the life cycle of the pathogen as well as the temporal and spatial development of general and focal late-blight epidemics for various scales and patterns of host genotypes. The novel contribution of this model is its ability to capture spatial relationships in the potato late blight pathosystem, and Figure 2 illustrates how the spatial development of potato late blight epidemics can be classified on a scale ranging from focal to general depending on the spatial distribution of primary infections. Spatial relationships are implemented in the model using a twodimensional negative exponential (radial Laplace) kernel for the

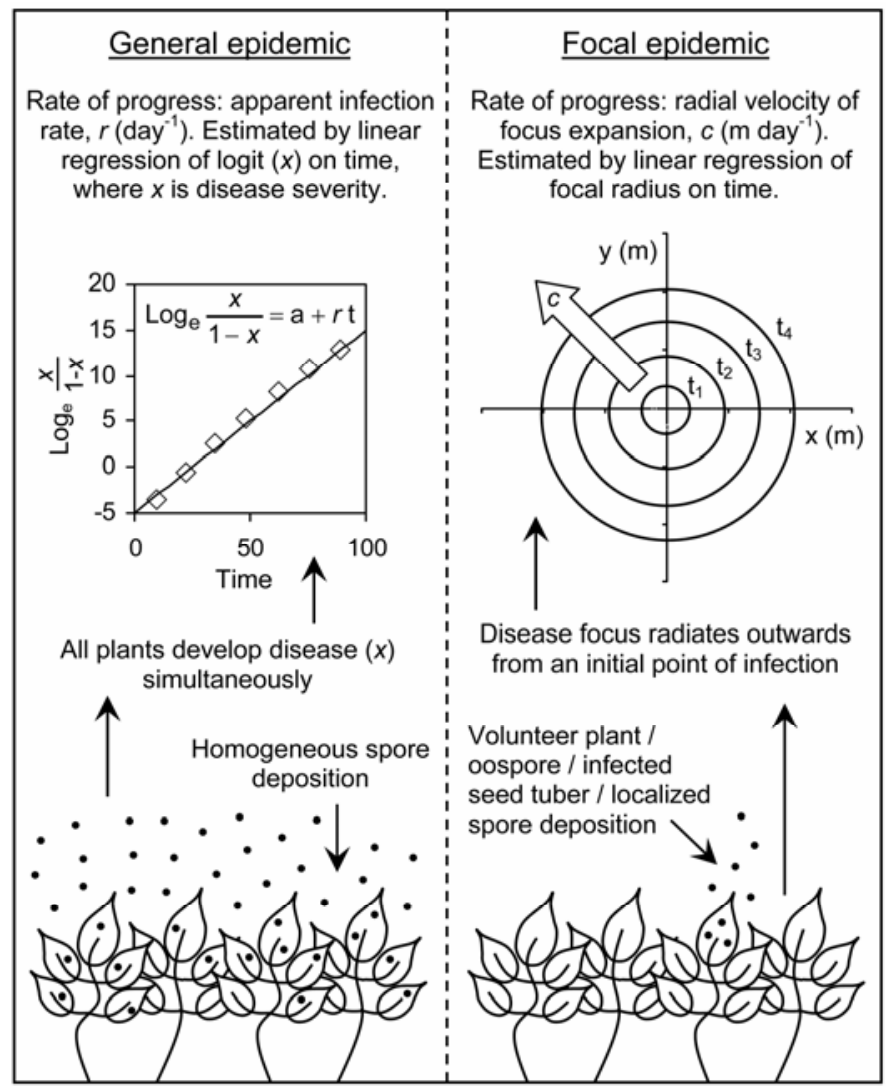

Fig. 2. Illustration of the differences between general and focal potato late blight epidemics and how the rate of epidemic progress is calculated for both epidemic types in the model. 
dispersal of spores $(16,17)$. Parameter values for the kernel used here were taken from Paysour and Fry (13), where experimental data were used to develop and validate a negative exponential kernel for the dispersal of $P$. infestans sporangia. The model is intended as a research tool and was designed for generating and testing hypotheses relating to epidemiological theory; more specifically, the influence of host diversity on the development of epidemics. Recently, the original model was extended with weather relationships and laboratory experiments were used to derive quantitative components of resistance for a number of potato cultivar-isolate combinations (16). The model was then validated against observed epidemics from field trials with five potato cultivars and two isolates, conducted in Wageningen, the Netherlands in 2002 and 2004 (16). In this study, the model is extended to include the growth of the potato host plant and the influence of fungicide applications. As a research tool, the primary value of this model is heuristic; therefore, based on the principles of parsimony (20) and transparency, simple logistic-type equations are implemented as models for foliage growth and fungicide use, and a simple linear function of light interception describes tuber dry matter production. The most important extensions to the model are described below. Table 1 provides a summary of symbol definitions and units.

Weather data. In this study, historical weather data from the Meteorology and Air Quality section at Wageningen University and Research Center in Wageningen, the Netherlands were used (available online). These data sets covered a 10-year span: 1994 and 1996 to 2004 (data for 1995 were incomplete). The influence of climatic conditions on the pathosystem was characterized with hourly measurements of the following meteorological variables: temperature $\left({ }^{\circ} \mathrm{C}\right)$, global radiation (i.e., the sum of direct and diffuse radiation) $\left(\mathrm{MJ} \mathrm{m}^{-2} \mathrm{~h}^{-1}\right)$, and relative humidity (\%).

Crop model. In this study, two levels of cultivar resistance are combined with two types of maturity class. Resistance level affects the growth rate of epidemics while maturity class determines the growth duration of the crop and, hence, the period of time the epidemic can grow. Although late-maturing cultivars are generally more resistant in practice than early-maturing cultivars, resulting in a confounding of the effects of maturity type on epidemic development and time available for the pathogen to cause damage, the effects of maturity type and resistance level can be studied in isolation in a modeling framework. This separation of resistance level and growth duration in the scenario studies allows greater insight into which factors are responsible for crop response to cultivar traits in relation to infection by $P$. infestans. Cultivar-isolate specific interactions are characterized in the model using three quantitative components of resistance: infection efficiency $(1,-)$, radial lesion growth rate $\left(\rho, \mathrm{m} \mathrm{day}^{-1}\right)$, and sporulation intensity $\left(\sigma\right.$, sporangia $\left.\mathrm{m}^{-2}\right)$. These were measured on potato leaflets in the laboratory, providing parameter values for a susceptible and resistant cultivar (16). The latent period $(\lambda$, days) is fixed at 5 days and the infectious period (I, days) at 1 day for all cultivars. The model has been validated against observed epidemics using these parameter values (16). General growth characteristics of early- and late-maturing cultivars were described using parameter values that lie well within the generally accepted range for these cultivar types. A complete listing of model parameters for simulation of different cultivars is given in Table 2 .

Leaf area development is simulated through thermal time accumulation by computing the growing degree days, $t_{d}\left({ }^{\circ} \mathrm{C}\right.$-day), as the positive difference between the (average daily) temperature and a base temperature of $2^{\circ} \mathrm{C}$ below which leaf growth stops (22). Early- and late-maturing cultivars have a different maximum leaf area index, $L(-)$, but the same relative growth and death rates. Leaf area is initialized at $155 \mathrm{~cm}^{2}$ plant $^{-1}(18)$, and leaf area expansion is calculated as a function of $t_{d}$ according the Richards equation (14):

$$
\frac{d L}{d t_{d}}=r_{g} L\left[1-\left(\frac{L}{L_{\max }}\right)^{\alpha}\right]
$$

TABLE 1. Symbols used in this study

\begin{tabular}{|c|c|c|}
\hline Symbol & Units $^{\mathrm{a}}$ & Description \\
\hline$f_{h}$ & - & Fraction of healthy tissue \\
\hline$k_{\mathrm{l}}$ & - & Light extinction coefficient \\
\hline$\ell$ & _ & Relative yield loss \\
\hline$L_{\max }$ & - & Maximum attainable leaf area index \\
\hline$r_{d}$ & ${ }^{\circ} \mathrm{C}$-day ${ }^{-1}$ & Relative death rate \\
\hline$r_{g}$ & ${ }^{\circ} \mathrm{C}$-day ${ }^{-1}$ & Relative growth rate \\
\hline$R_{g}$ & $\mathrm{MJ} \mathrm{m}^{-2}$ & Daily amount of incoming global radiation \\
\hline$t$ & Days & Time \\
\hline$t_{a}$ & Days & Time of the last fungicide application \\
\hline$t_{d}$ & ${ }^{\circ} \mathrm{C}$-day & Growing degree days \\
\hline$t_{50}$ & Days & Time after a fungicide application at which $\mathrm{t}$ reaches half of its maximum value \\
\hline$u$ & $\mathrm{~m} \mathrm{~s}^{-1}$ & Wind speed \\
\hline$v_{d}$ & $\mathrm{~m} \mathrm{~s}^{-1}$ & Deposition velocity \\
\hline$Y$ & Tons (DM) ha- ${ }^{-1}$ & Final yield \\
\hline$\varepsilon$ & - & Escape fraction \\
\hline 1 & - & Infection efficiency \\
\hline $\mathrm{t}_{\max }$ & - & Maximum infection efficiency \\
\hline$i_{\min }$ & - & Minimum infection efficiency \\
\hline I & Days & Infectious period \\
\hline$\kappa$ & - & von Kármán constant $(0.35)$ \\
\hline$\lambda$ & Days & Latent period \\
\hline$\rho$ & $\mathrm{m} \mathrm{day}^{-1}$ & Lesion growth rate \\
\hline$\sigma$ & $\mathrm{m}^{-2}$ & Sporulation intensity \\
\hline$v$ & $\mathrm{~g} \mathrm{MJ}^{-1}$ & Light use efficiency \\
\hline
\end{tabular}

${ }^{a}$ Dash (-) signifies that the defined quantity is dimensionless. 
where $r_{g}\left({ }^{\circ} \mathrm{C}\right.$-day $\left.{ }^{-1}\right)$ is the relative leaf growth rate during exponential growth, $L_{\max }(-)$ is the maturity class specific maximum attainable leaf area index, and $\alpha(-)$ is a shape factor used to control the speed at which the function approaches its plateau and, thus, the shape of the growth curve. The shape parameter is used to ensure that both early- and late-maturing cultivars require the same amount of time to reach a fully developed canopy. In accordance with the model of van Oijen (23), $r_{g}$ is set to $0.02{ }^{\circ} \mathrm{C}$ day $^{-1}$ for all cultivars. Degree days accumulate over time to provide a temperature sum, and leaf area will continue to grow until the temperature sum reaches a maximum cultivar-specific value (estimated as $500{ }^{\circ} \mathrm{C}$-days for early-maturing cultivars and $1,000{ }^{\circ} \mathrm{C}$-days for late-maturing cultivars), at which point net leaf growth becomes net leaf death and the shedding of leaves follows a logistic function of $t_{d}$ :

$$
\frac{d L}{d t_{d}}=-r_{d} L\left(1-\frac{L}{L_{\max }}\right)
$$

where $r_{d}\left({ }^{\circ} \mathrm{C}\right.$-day $\left.{ }^{-1}\right)$ is the relative leaf death rate, set in accordance with van Oijen (23) to $0.01{ }^{\circ} \mathrm{C}^{- \text {day }^{-1}}$ for all cultivars. Leaf area index is maintained near its maximum value for some time before leaf shedding begins, as is typical of growth patterns occurring in the field. Shedding affects all tissue types equally (i.e., shedding results in equal, proportional loss of healthy, latent, infectious, and necrotic areas). Green leaf area duration is reduced not only by the coverage of leaf area by lesions but also because lesions can girdle stems and cause additional necrotic areas in noninfected leaf tissue; during the epidemic, an increase in latent area is assumed to cause an equal increase in the loss of the nonlesion-covered leaf area $(16,23)$. Leaf lesion coverage and areas of necrotic leaf tissue caused by $P$. infestans are assumed to be distributed homogeneously throughout the canopy.

Under the assumption of equal profiles of healthy and blighted leaf tissue over canopy depth, the interception of solar radiation is calculated using Lambert-Beer's law:

$$
R_{L}=f_{h} R_{g}\left[1-\exp \left(-k_{1} L\right)\right]
$$

where $R_{L}\left(\mathrm{MJ} \mathrm{m}^{-2}\right)$ is daily intercepted radiation, $f_{h}(-)$ is the fraction of healthy tissue, $R_{g}\left(\mathrm{MJ} \mathrm{m}^{-2}\right)$ is the daily incoming global radiation, and $k_{1}(-)$ is the light extinction coefficient, set to 1 (7). $R_{L}$ is integrated over time and multiplied with the average light use efficiency for tuber dry matter production to give the tuber dry weight of the crop (6):

$$
Y=v R_{L, c} \times 10^{-2}
$$

where $Y$ (tons $\left.[\mathrm{DM}] \mathrm{ha}^{-1}\right)$ is tuber yield, $v\left(\mathrm{~g} \mathrm{MJ}^{-1}\right)$ is the light use efficiency for tuber dry matter and global radiation, $R_{L, c}\left(\mathrm{MJ} \mathrm{m}^{-2}\right)$ is the cumulative intercepted global radiation, and the final term is a conversion factor. On the basis of experimental results, early cultivars were assigned light use efficiencies of $1.1 \mathrm{~g} \mathrm{MJ}^{-1}$ and late cultivars $1.0 \mathrm{~g} \mathrm{MJ}^{-1}$ (23).

Leaf area index also determines the fraction of spores that can escape the potato canopy and be made available for long-range transport; the remaining fraction is assumed to be deposited back onto the crop to contribute to local epidemic development. The mechanistically derived model of de Jong et al. (3) is used to provide an estimate of the escape fraction, $\varepsilon(-)$, as a function of $L$, the deposition velocity of $P$. infestans sporangia, $v_{d}\left(\mathrm{~m} \mathrm{~s}^{-1}\right)$, and wind speed, $u\left(\mathrm{~m} \mathrm{~s}^{-1}\right)$. The remaining fraction is used to define the deposition efficiency, $\delta(-)$, of spores back onto the crop:

$$
\delta=1-\varepsilon=1-\exp \left[-L \sqrt{v_{d} /(\kappa u)}\right]
$$

where $\kappa(-)$ is the von Kármán constant (0.35). According to Gregory (5), a settling velocity for $P$. infestans sporangia of $0.0085 \mathrm{~ms}^{-1}$ and a deposition velocity equal to three times the settling velocity is assumed, giving a fixed value of $0.0255 \mathrm{~m} \mathrm{~s}^{-1}$ for $v_{d}$. A constant wind speed of $2 \mathrm{~m} \mathrm{~s}^{-1}$ is also assumed to be representative of wind speed at crop height (during dispersal events) in the Netherlands.

Fungicide model. Late blight management is simulated with protectant fungicides, which decrease the infection efficiency, 1 (-), of sporangia. As an alternative to modeling the concentration of the active ingredient on the leaf, a Weibull function is used to simulate the application and decay of fungicides via their effect on $\mathrm{t}$ :

$$
\begin{array}{cr}
\mathrm{l}=\mathrm{l}_{\text {min }} & t=t_{a} \\
\mathrm{l}=\mathrm{l}_{\text {min }}+\frac{\mathrm{l}_{\text {max }}-\mathrm{l}_{\text {min }}}{1+\left[t_{50} /\left(t-t_{a}\right)\right]^{\beta}} & t>t_{a}
\end{array}
$$

where $\imath_{\min }(-)$ is the value $\imath$ drops to immediately after an application, $t$ (days) is time, $t_{a}$ (days) is the time of the last fungicide application, $\mathfrak{l}_{\max }(-)$ is the maximum value for the cultivar, $t_{50}$ (days) is the time after application at which $\mathrm{l}$ is halfway restored after the drop caused by the application, and $\beta(-)$ is a shape factor. Parameter values were chosen so as to represent the effects (level of protection and typical decay rate) of Shirlan (a.i., Fluazinam) on the leaves: $\boldsymbol{l}_{\min }$ is set to $1 \%$ of $\mathbf{l}_{\max }, t_{50}$ is set to 9 days, and $\beta$ is set to $20($ H. T. A. M. Schepers, personal communi-

\begin{tabular}{|c|c|c|c|c|}
\hline \multirow[b]{2}{*}{ Parameters } & \multicolumn{4}{|c|}{ Cultivar types } \\
\hline & Early & Late & Susceptible & Resistant \\
\hline \multicolumn{5}{|l|}{ Maturity class } \\
\hline Date of emergence & 1 May & 1 May & $\ldots$ & $\ldots$ \\
\hline Date of harvest & 31 July & 30 September & $\ldots$ & $\ldots$ \\
\hline Onset of leaf shedding $\left({ }^{\circ} \mathrm{C}\right.$-day) & 500 & 1,000 & $\ldots$ & $\ldots$ \\
\hline Tuber initiation $\left({ }^{\circ} \mathrm{C}\right.$-day) & 150 & 350 & $\ldots$ & $\ldots$ \\
\hline$L_{\max }(-)$ & 3 & 6 & $\ldots$ & $\ldots$ \\
\hline$\alpha(-)$ & 1 & 0.6 & $\ldots$ & $\ldots$ \\
\hline$r_{g}\left({ }^{\circ} \mathrm{C}-\mathrm{day}^{-1}\right)$ & 0.02 & 0.02 & $\ldots$ & $\ldots$ \\
\hline$r_{d}\left({ }^{\circ} \mathrm{C}-\mathrm{day}^{-1}\right)$ & 0.01 & 0.01 & $\ldots$ & $\ldots$ \\
\hline$v\left(\mathrm{~g} \mathrm{MJ}^{-1}\right)$ & 1.1 & 1.0 & $\ldots$ & $\ldots$ \\
\hline \multicolumn{5}{|l|}{ Level of partial resistance } \\
\hline $\mathrm{l}(-)$ & $\ldots$ & $\ldots$ & $3.12 \times 10^{-2}$ & $1.00 \times 10^{-2}$ \\
\hline I (days) & $\ldots$ & $\ldots$ & 1 & 1 \\
\hline$\lambda$ (days) & $\ldots$ & $\ldots$ & 5 & 5 \\
\hline$\rho\left(\mathrm{m} \mathrm{day}^{-1}\right)$ & $\ldots$ & $\ldots$ & $4.76 \times 10^{-3}$ & $1.70 \times 10^{-3}$ \\
\hline$\sigma\left(\mathrm{m}^{-2}\right)$ & $\ldots$ & $\ldots$ & $4.55 \times 10^{8}$ & $1.64 \times 10^{8}$ \\
\hline
\end{tabular}
cation). The infection efficiency calculated with equation 6 is then adjusted to account for the growth of new, unprotected leaves, which further reduce the efficacy of the fungicide treatment:

TABLE 2. Model parameters for potato cultivar types distinguished in the study 


$$
\mathfrak{l}=\min \left\{\mathrm{l}_{\max }, \frac{L 1+\Delta L 1_{\max }}{L+\Delta L}\right\}
$$

where $\Delta L(-)$ is the increase in leaf area index since the last application of fungicides.

Simulation. Space is represented explicitly as a two-dimensional grid, in which each cell represents a potato plant. Host plants are linked through dispersal of spores and we describe where spores land with a radial Laplace dispersal kernel, which describes the probability distribution of landing locations of spores in the two-dimensional plane $(16,17)$. As described by Skelsey et al. $(16,17)$, spatial phenomena in the model are solved by performing convolutions between spatial distributions of spores and dispersal kernels with the aid of fast Fourier transforms. Based upon planting patterns in the Netherlands, the length of the side of a compartment cell is set at $0.75 \mathrm{~m}$ (distance between rows) and the width of each compartment cell at $0.3 \mathrm{~m}$ (distance between plants). In each simulation experiment, the field size is fixed at 76.8 by $48 \mathrm{~m}$ and contains a pure-line population of one of the four cultivar types. Each field is isolated in the sense that it is completely surrounded by noncrop area. Thus, apart from the initial inoculum, there is no further influx of spores into the field. On the other hand, according to the dispersal kernel, $\approx 5 \%$ of the spores produced in the field are dispersed outside the field boundaries to the surrounding noncrop area. These spores do not contribute to epidemic progress. Simulations are stopped when the overall percentage of visibly diseased (sporulating plus necrotic) foliage reaches $5 \%$, in order to simulate compulsory destruction of the haulm according to Dutch directives for Phytophthora spp. control.

Final yield is chosen as the model output of relevance in this study because it is the relevant criterion in decision support. $P$. infestans sporangia can infect both foliage and tubers directly; however, it was not possible to incorporate tuber infection in this study because there is insufficient data with which to model the process. In order to assess the influence of spore load on final yield, general and focal epidemics are simulated in each of the four cultivar types and for four different fungicide regimes. The number of spores that arrive at a target crop and initiate an epidemic depends on several factors, such as distance and air flow between source and target, the number of spores released at the source, the dilution of the spore cloud by the combined effects of advection and turbulence, and the loss of spores by deposition and death during flight (1). Fifteen different levels of initial spore load are used as input data: $0,1 \times 10^{0}, 3 \times 10^{0}, 1 \times 10^{1}, 3 \times 10^{1}, 1 \times$ $10^{2}, 3 \times 10^{2}, 1 \times 10^{3}, 3 \times 10^{3}, 1 \times 10^{4}, 3 \times 10^{4}, 1 \times 10^{5}, 3 \times 10^{5}$, $1 \times 10^{6}$, and $3 \times 10^{6}$ spores $\mathrm{m}^{-2}$. Logarithmic series are used because the relevant range of spore loads is large. These densities are translated to an equivalent spore dose per plant; general inoculation proceeds with the application of the appropriate spore dose to each plant in the field whereas, for focal inoculation, only a central three-by-three block of nine plants in the field receives this dose. Inoculation occurs once, on the first suitable day for potato late blight as determined by the weather relationships in the model. In this study, the initial spore load is assumed to come from a distant source of infection; thus, each simulation experiment begins with a nondiseased crop. In order to demonstrate that the selected range of initial spore loads used lies within the realm of possible spore fluxes that occur in nature, data from field experiments (2) were considered; aerial concentrations of $P$. infestans sporangia were measured directly above infected potato canopies and a maximum value of $\approx 30,000$ sporangia $\mathrm{m}^{-3}$ was recorded. Assuming a worst-case scenario in which all these spores were viable, this value can be considered to lie at the extreme limit of possible aerial concentrations of viable spores arriving at a target field. After multiplication with the deposition velocity of $P$. infestans sporangia, $0.0255 \mathrm{~m} \mathrm{~s}^{-1}$ (5), over an 8 -h release period, a flux density of $\approx 2 \times 10^{7}$ spores $\mathrm{m}^{-2}$ is arrived at, which is slightly higher than the maximum value of initial spore load used in this study. The four cultivar types used as target crops in this study were (i) early/resistant, (ii) early/susceptible, (iii) late/resistant, and (iv) late/susceptible. The fungicide regimes were as follows: (i) no applications; (ii) an adaptive regime based on the suitability of the weather for late blight, where the minimum interval between designated applications is 5 days; (iii) an adaptive regime as in ii except that the first application is missed; and (iv) a fixed schedule, where fungicides are applied every 7 days. This makes a total of 16 different management scenarios ( 4 cultivars $\times 4$ fungicide regimes) and 480 epidemic scenarios (16 management scenarios $\times 15$ spore fluxes $\times 2$ epidemic types: general and focal). In order to investigate the influence of variation in climatic conditions on model results, each epidemic scenario was repeated using a different meteorological data-set from the 10 years available. This makes a total of 4,800 simulation experiments (480 epidemic scenarios $\times 10$ years of meteorological data).

Data treatment. Model predictions for final yield in each of the 480 epidemic scenarios were averaged over the 10 years of meteorological input data. The resultant (averaged) final yields, $Y$ (tons $[\mathrm{DM}] \mathrm{ha}^{-1}$ ), were used to calculate the loss in yield relative to the disease-free yield:

$$
\ell=\frac{Y_{0}-Y}{Y_{0}}
$$

where $\ell(-)$ is the relative yield loss and $Y_{0}$ is the (average) final yield obtained with a disease-free crop. These data are presented on semi-log plots of relative yield loss against log of initial spore load (damage curves).

\section{RESULTS}

Three example epidemics, differing only in the initial amount of inoculum, are used in order to illustrate the mechanism by which an increase in initial spore load results in a reduction in final yield (Fig. 3). Lesions act independently of one another in the exponential phase of an epidemic; therefore, an increase in initial spore load only affects the starting density of the disease; it does not alter the relative growth rate of the epidemic or the shape of the disease progress curve. The increased spore input thus shifts the disease progress curve to the left on the time-axis (Fig. 3A). Earlier epidemic onset means that (according to Dutch regulations) farmers must destroy the haulm earlier in the season, and there is less time available for foliage growth (Fig. 3B). Less foliage means that less light can be intercepted (Fig. 3C), resulting in a decrease in the production and allocation of photosynthetic products to the tubers (Fig. 3D).

A frequency histogram is given to show the number of infection events in each of the 10 growing seasons, as determined by observed weather in Wageningen (1994 and 1996 to 2004) in combination with the weather relationships (Fig. 4). Also shown is the number of fungicide applications under the adaptive fungicide regime (regime 2) in each growing season. The highest number of infection events in any year was 23 (1994 and 2001), the lowest number was 7 (1996), and the average over the 10 years was 15 . It can be seen that the number of infection events differs widely between years, illustrating the importance of epidemic simulation over a wide and representative diversity of conditions. The maximum number of fungicide applications in any year was 12 (1994 and 2000), the minimum number was 5 (1996), and the average number of applications per growing season was 9 . This is in stark contrast to the 20 applications that were simulated under the 7-day fixed-spray schedule (regime 4).

Predicted damage curves are grouped for resistant and susceptible cultivars (Figs. 5 and 6, respectively). The simulated late blight epidemics were highly variable, with lower relative yield 
loss values for early- as opposed to late-maturing cultivars and resistant as opposed to susceptible cultivar types. Relative yield loss values (taken over all fungicide regimes and inoculation types) for the early/resistant cultivar type ranged from a minimum of 0 to a maximum of 0.91 , depending on initial spore load, with a mean loss of 0.19 when averaged over initial spore loads, and a standard deviation of 0.29 . The range and spread of values was similar for the early/susceptible cultivar type, although the aver- age loss was much higher, at 0.42 . For the late/resistant cultivar type, relative yield loss values ranged from 0 to 0.97 , depending on initial spore load, with a mean loss of 0.35 when averaged over initial spore loads, and a standard deviation of 0.33 . The late/ susceptible cultivar type sustained the heaviest losses of the four cultivar types; relative yield loss values ranged from a minimum of 0.27 to a maximum of 0.97 , with a mean loss of 0.68 and a standard deviation of 0.2 .
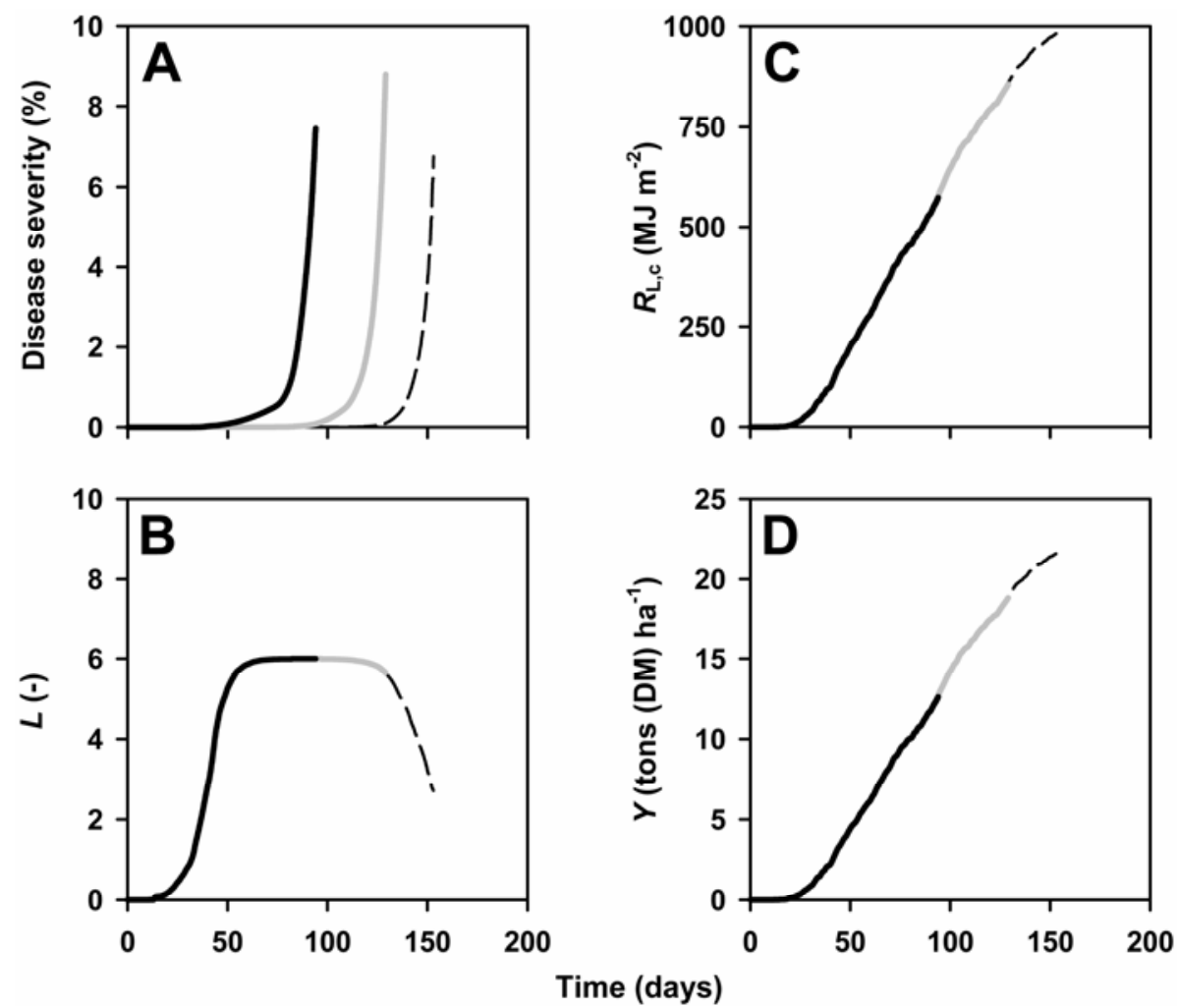

Fig. 3. Mechanism by which an increase in initial spore load (three line types) results in a decrease in final yield in the spatiotemporal model of the potato late bight pathosystem: A, epidemic increase; $\mathbf{B}$, leaf area dynamics of crop (leaf area index - infected and green tissue); $\mathbf{C}$, cumulative radiation interception by crop; and $\mathbf{D}$, dry matter accumulation in tubers. Higher levels of initial spore load are indicated by lines of increasing intensity.

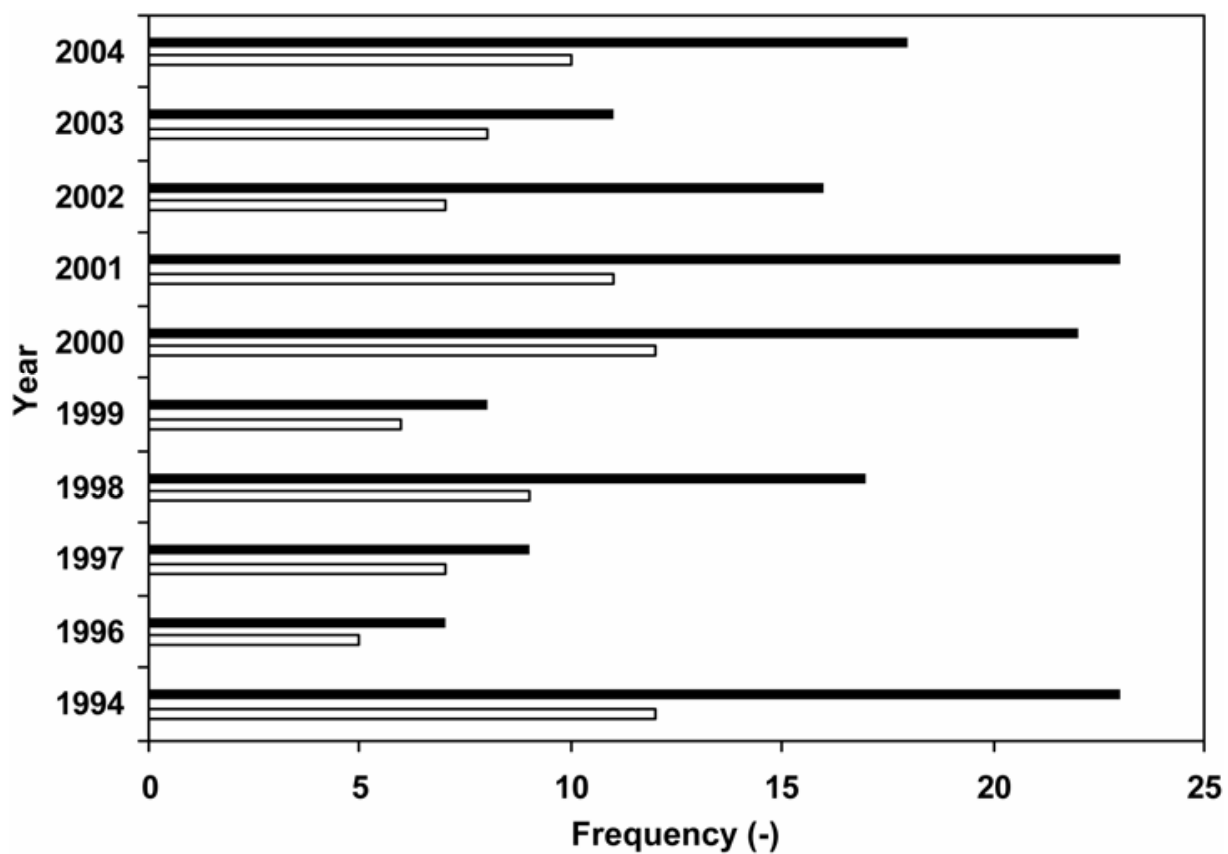

Fig. 4. Number of infection events (solid bars) and fungicide applications (open bars) under an adaptive spray regime (regime 2) in 10 growing seasons (1 May to 30 September), as calculated by the spatiotemporal model of the potato late bight pathosystem using weather data from Wageningen University Meteostation in the Netherlands. 
Relative yield loss increased more rapidly in response to changes in initial spore load when the spatial distribution of initial inoculum was homogeneous (general epidemic) as opposed to focal (focal epidemics). This is because few plants are acting as initial sources of infection in the field in a focal epidemic, in contrast to general epidemics where every plant in the crop is an initial source. Therefore, a focal epidemic must progress in time and space before the entire field is infected whereas, in a general epidemic, increase in disease is essentially temporal. Early cultivar types were less responsive to changes in initial spore load than late cultivar types, because early-maturing cultivars escape part of the epidemic by completing a greater fraction of their tuber filling before the disease causes premature net foliage death. Susceptible cultivars responded with greater yield losses than resistant cultivars, and an initial spore load of 1 spore $\mathrm{m}^{-2}$ was enough to result in yield loss in all susceptible management scenarios. The slope of the damage curve was generally shallower for susceptible cultivars as a result of relatively large initial yield losses at the lowest level of spore input tested. A further explanation is that initial differences in resistance level and yield response diminished as spore loads became increasingly overwhelming. The adaptive fungicide regime (regime 2) was always the most effective of the four simulated regimes because applications always occurred immediately prior to the deposition of primary inoculum (and any subsequent infection event, provided the minimum spray interval had been exceeded). However, the adaptive regime was not capable of preventing yield loss under high initial spore loads, because fungicide protection is not absolute. Under focal inoculation (Figs. 5 and 6, panels $\mathrm{A}$ and $\mathrm{C}$ ), an adaptive fungicide regime with the first spray of the season missed (regime 3) always provided a higher level of protection than a weekly spray regime (regime 4). The reverse was true under general inoculation because every plant in the crop left unprotected subsequently became infected (Figs. 5 and 6, panels B and D).

Interestingly, under focal inoculation, an increase in initial spore load did not always lead to an increase in relative yield loss. This is a demonstration of nonlinear effects in the onset of epidemics and their interaction with weather in the model; primary foci can burn out if the weather prevents propagation of lesions and all available healthy tissue is consumed by lesion expansion.

The steepness of the damage curves indicates the degree of importance that would be attached to obtaining accurate knowledge on dispersal for a practical DSS (Figs. 5 and 6). Whenever fungicides were used in conjunction with a resistant cultivar, damage curves were generally flat up to a level of several hundred spores per square meter, sometimes as high as tens of thousands of spores per square meter for focal epidemics. This lack of response to spore input indicates resilience to inaccuracies in forecasts of spore inputs, suggesting that dispersal information

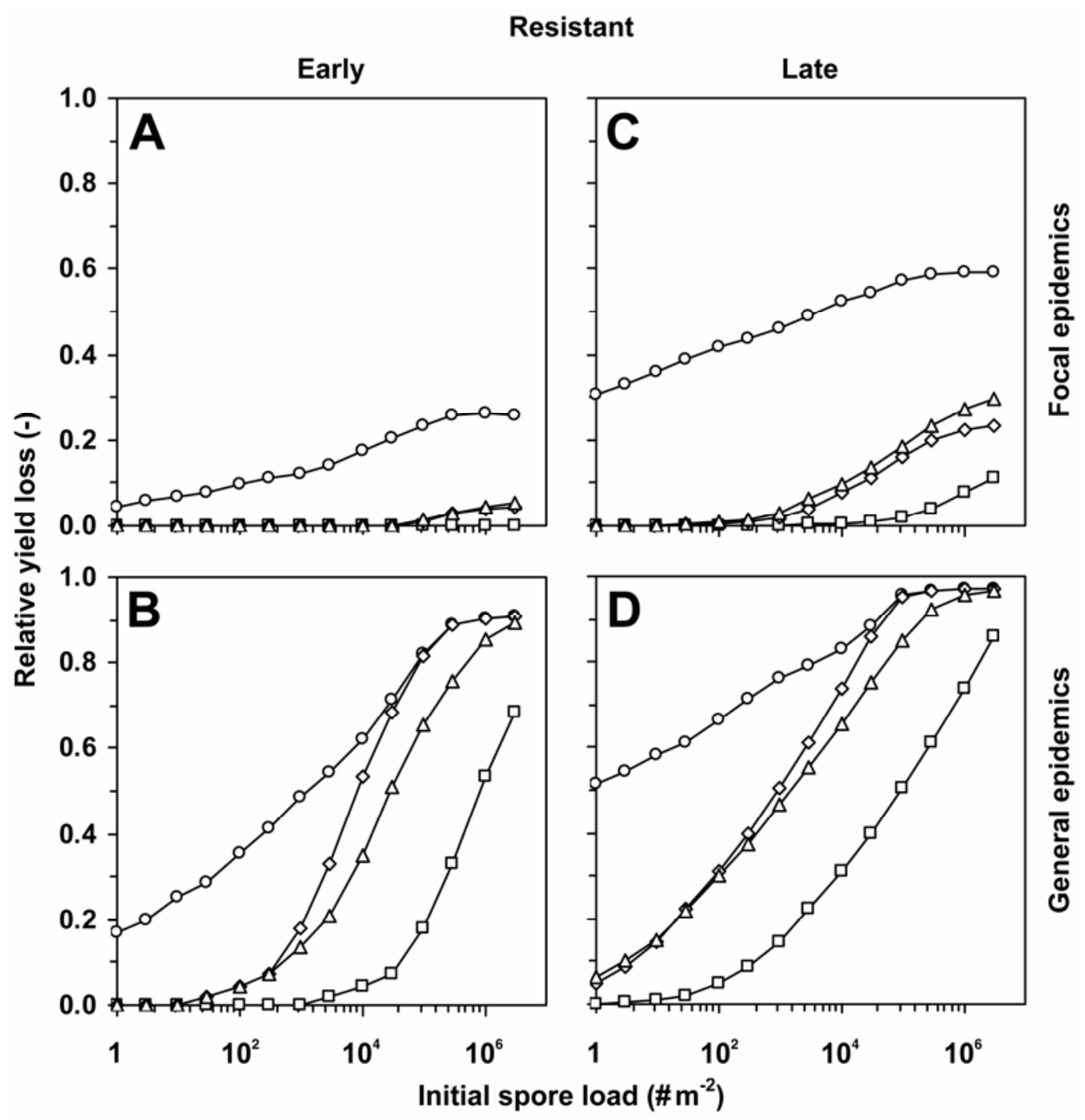

Fig. 5. Relationship between yield loss (relative to final yield in a disease-free crop) and primary inoculum (initial spore load) in simulated potato late blight epidemics in resistant cultivars. A and C, Focal epidemics; B and D, general epidemics; $\mathbf{A}$ and B, early-maturing cultivar; $\mathbf{C}$ and $\mathbf{D}$, late-maturing cultivar. In each panel, four fungicide regimes are compared: $\square=$ adaptive; $\diamond=$ adaptive with the first spray missed, $\Delta=7$-day fixed schedule; and $O=$ no applications. 
could be used in decision support where resistant cultivars are concerned without an undue increase in the risk of yield loss to the grower if the potential for spore dispersal between fields was underestimated. Susceptible cultivars, and especially susceptible late cultivars, suffered sizeable yield losses even at the lowest tested spore input of 1 spore $\mathrm{m}^{-2}$. This indicates that it would be risky to place a heavy emphasis on dispersal information or dispersal models for management decisions with susceptible cultivars, because an unrealistic level of accuracy would be required.

\section{DISCUSSION}

The scenario-based approach presented in this article was used to determine the scope for inclusion of spatial information (i.e., dispersal information and dispersal modeling) in DSSs for aerially transmitted pathogens. With specific regard to the model pathogen (P. infestans), the results of this study provide valuable information for potato late blight initiatives such as the Umbrella Plan Phytophthora in the Netherlands, which is part of a covenant between the Dutch Ministry of Agriculture and a grower organization, Land- en Tuinbouw Organisatie. The aim of this particular initiative is to diminish the dependency on fungicides in the shortterm via an increase in the efficacy of chemical treatments and improvements in DSSs and, in the long term, via the development of resistant cultivars. One of the objectives of our research is to determine whether atmospheric dispersion and spore survival models could be used to modify the spray recommendations of existing DSSs. Currently, almost all DSSs work on the assumption that conducive weather for disease equates to the arrival of viable inoculum at all target crops. It is possible that new decision rules that consider the effects of weather on long-distance dispersal and survival of spores could prove to be effective in reducing the number of chemical applications in a growing season. This study indicates that, for susceptible cultivars, any DSS that places emphasis on the dispersal capabilities of the pathogen would require dispersal information at a level of accuracy that is unlikely to be practically attainable. The probability of making an inappropriate spray decision would be high and the consequences to the grower could be extensive. In contrast, the relative tolerance of resistant cultivars to primary inoculum means that there is room for uncertainty in dispersal information and the probability of making an inappropriate spray decision (with consequences) is greatly decreased. These results indicate that there exists scope for including spore load estimations in DSSs for potato late blight for resistant potato cultivars but not for susceptible cultivars.

The contribution of this article is to present a scenario-based simulation approach that can be used to assess ex ante the use of dispersal information in the development and evaluation of DSSs for aerially transmitted pathogens. This approach was used with a model pathogen ( $P$. infestans) to evaluate the range of spore loads

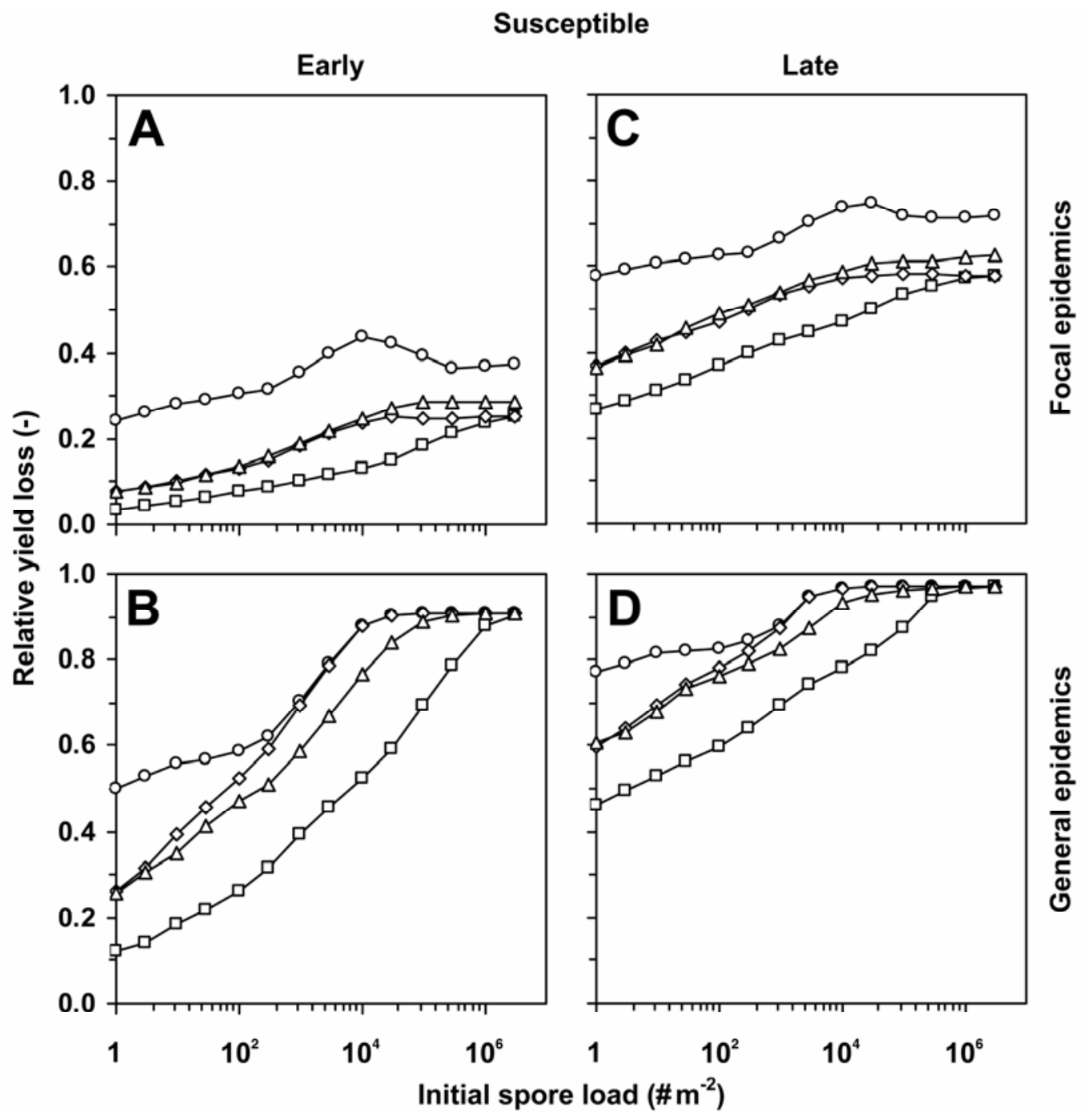

Fig. 6. Relationship between yield loss (relative to final yield in a disease-free crop) and primary inoculum (initial spore load) in simulated potato late blight epidemics in susceptible cultivars. A and C, Focal epidemics; $\mathbf{B}$ and $\mathbf{D}$, general epidemics; $\mathbf{A}$ and $\mathbf{B}$, early-maturing cultivar; $\mathbf{C}$ and $\mathbf{D}$, late-maturing cultivar. In each panel, four fungicide regimes are compared: $\square=$ adaptive; $\diamond=$ adaptive with the first spray missed, $\Delta=7$ day fixed schedule; and $\bigcirc=$ no applications. 
that pose a threat to potato crops. Model results demonstrated an important effect of cultivar-management decisions on yield loss associated with a particular spore load, and enabled an evaluation of the potential for inclusion of dispersal information in decision support for potato late blight in terms that matter to growers. The development and experimental validation of a DSS for potato late blight that has a regional aerobiological component is the subject of a subsequent study (15).

\section{ACKNOWLEDGMENTS}

Funding for this study was provided by the Dutch Ministry of Agriculture, Nature Management and Fisheries through the Umbrella Plan Phytophthora (DWK 427).

\section{LITERATURE CITED}

1. Aylor, D. E. 1986. A framework for examining inter-regional aerial transport of fungal spores. Agric. For. Meteorol. 38:263-288.

2. Aylor, D. E. 2001. Quantifying the rate of release and escape of Phytophthora infestans sporangia from a potato canopy. Phytopathology 91:11891196.

3. de Jong, M. D., Bourdôt, G. W., Powell, J., and Goudriaan, J. 2002. A model of the escape of Sclerotinia sclerotiorum ascospores from pasture. Ecol. Model. 150:83-105.

4. Ferrandino, F. J. 1989. A distribution-free method for estimating the effect of aggregated plant damage on crop yield. Phytopathology 79:1229-1232.

5. Gregory, P. H. 1973. The Microbiology of the Atmosphere, 2nd ed. John Wiley \& Sons, New York.

6. Haverkort, A. J., and Harris, P. M. 1987. A model for potato growth and yield under tropical highland conditions. Agric. For. Meteorol. 39:271282.

7. Haverkort, A. J., and Kooman, P. L. 1997. The use of systems analysis and modeling of growth and development in potato ideotyping under conditions affecting yields. Euphytica 94:191-200.

8. Haverkort, A. J., Top, J., and Verdenius, J. 2006. Organizing data in arable farming: towards an ontology of processing potato. Pot. Res. 49:177-201.

9. James, W. C. 1974. Assessment of plant diseases and losses. Annu. Rev. Phytopathol. 12:27-48.

10. Jeger, M. J. 1999. Improved understanding of dispersal in crop pest and disease management: Current status and future directions. Agric. For. Meteorol. 97:331-349.

11. Madden, L. V., Hughes, G., and van den Bosch, F. 2007. The Study of Plant Disease Epidemics. The American Phytopathological Society, St. Paul, MN.

12. Madden, L. V., Pennypacker, S. P., Antle, C. E., and Kingsolver, C. H. 1981. A loss model for crops. Phytopathology 71:685-689.

13. Paysour, R. E., and Fry, W. E. 1983. Interplot interference: A model for planning field experiments with aerially disseminated pathogens. Phytopathology 73:1014-1020.

14. Richards, F. J. 1959. A flexible growth function for empirical use. J. Exp. Bot. 10:290-300.

15. Skelsey, P., Kessel, G. J. T., Holtslag, A. A. M., Moene, A. F., and van der Werf, W. 2009. Regional spore dispersal as a factor in disease risk warnings for potato late blight: a proof of concept. Agric. For. Meteorol. 149:419-430.

16. Skelsey, P., Kessel, G. J. T., Rossing, W. A. H., and van der Werf, W. 2009. Parameterization and evaluation of a spatiotemporal model of the late blight pathosystem. Phytopathology 99:290-300.

17. Skelsey, P., Rossing, W. A. H., Kessel, G. J. T., Powell, J., and van der Werf, W. 2005. Influence of host diversity on development of epidemics: An evaluation and elaboration of mixture theory. Phytopathology 95:328338.

18. Spitters, C. J. T., van Keulen, H., and van Kraalingen, D. W. G. 1989. A simple and universal crop growth simulator: SUCROS87. Pages 147-181 in: Simulation and Systems Management in Crop Protection. R. Rabbinge, S. A. Ward, and H. H. van Laar, eds. Pudoc, Wageningen, The Netherlands.

19. Teng, P. S. 1987. Quantifying the relationship between disease intensity and yield loss. Pages 105-113 in: Crop Loss Assessment and Pest Management. P. S. Teng, ed. American Phytopathological Society, St. Paul, MN.

20. Turchin, P. 2003. Complex Population Dynamics: A Theoretical/ Empirical Synthesis. Princeton University Press, Princeton, NJ.

21. van der Plank, J. E. 1963. Plant Diseases: Epidemics and Control. Academic Press Inc., New York.

22. van Keulen, H., and Stol, W. 1995. Agro-ecological zoonation for potato production. Pages 357-371 in: Ecology and Modeling of Potato Crops Under Conditions Limiting Growth. A. J. Haverkort and D. K. L. Mackerron, eds. Kluwer, Dordrecht, The Netherlands.

23. van Oijen, M. 1992. Evaluation of breeding strategies for resistance and tolerance to late blight in potato by means of simulation modelling. Neth. J. Plant Pathol. 98:3-11. 